\title{
Ticked off basophils end banquet
}

Ticks are blood-feeding parasites that can transmit diseases, such as viral encephalitis, Lyme disease and babesiosis, during blood meals; therefore the development of effective strategies for tick control is an important public health issue. Immune resistance to ticks can occur after consecutive infestations but the mechanisms responsible are poorly understood. Now, by generating a basophil-specific conditional knockout mouse strain, Karasuyama and colleagues have shown an important role for basophils in acquired immunity to ticks.

Although mast cells and basophils are thought to be involved in tickspecific immunity, data from previous studies have been conflicting; the authors therefore set out to clarify the roles of both cell populations. Mice infested with the tick Haemaphysalis longicornis showed resistance 14 days after a secondary infestation, but acquired resistance was not seen in

mast cell-deficient mice. Previous

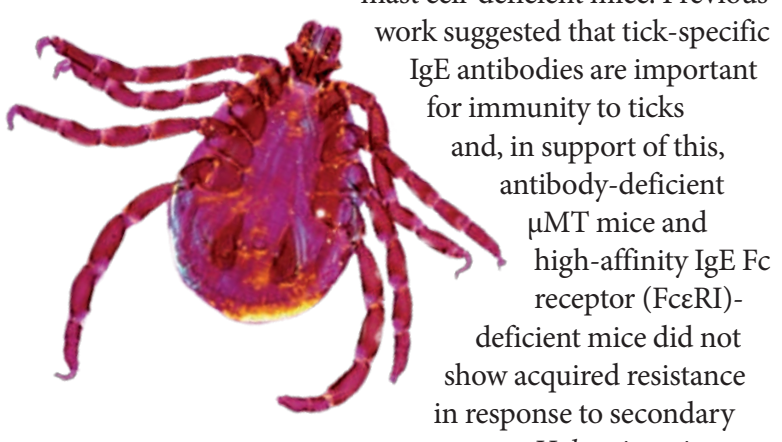

exposure to $H$. longicornis.

Surprisingly, mast cell-deficient mice that had been reconstituted with mast cells from FceRI-deficient mice showed normal acquired resistance, indicating that immunity to ticks is not dependent on IgE-mediated mast cell functions.

As the expression of FceRI is restricted to mast cells and basophils, these data suggested that basophils might also be involved in immunity to ticks. Analyses of skin tissue sections at various time points after tick exposure showed that basophils were rarely present during the primary infestation, but infiltrated the skin and clustered around tick mouths following secondary infestation. Furthermore, antibody-mediated depletion of basophils abolished acquired resistance to secondary infestation with $H$. longicornis but had no effect on primary tick infestation.

One problem with antibodymediated basophil depletion approaches is that the antibodies used can also react with mast cells. Therefore, to target basophils more specifically, the authors used the promoter for a basophil-specific protease, mast cell protease 8 (Mcpt8), to generate mice in which the expression of the diphtheria toxin receptor (DTR) is restricted to basophils (Mcpt8 ${ }^{D T R}$ mice). Accordingly, treatment of $M c p t 8^{D T R}$ mice with diphtheria toxin resulted in transient depletion of basophils but had no effect on other immune cells, including peritoneal and dermal mast cells. In addition, mast cell-dependent allergic responses in the skin were normal in diphtheria toxin-treated $M c p t 8^{D T R}$ mice, indicating that these animals had an intact and functional mast cell compartment. However, diphtheria toxin treatment abolished resistance to secondary tick infestation in the $M c p t 8^{D T R}$ mice, thereby confirming the importance of basophils for acquired tick-specific immune resistance.

Finally, the authors showed that transfer of basophils from previously infested mice could confer tick resistance to naive animals. However, acquired resistance was not observed if basophils from infested FceRIdeficient mice were transferred to naive recipients. These experiments confirm that IgE-mediated activation of basophils, rather than mast cells, is important for acquired resistance to ticks. Future studies addressing how basophils and mast cells cooperate to provide immunity to ticks could aid the development of novel strategies for the control of tick-borne disease. Yvonne Bordon 